\title{
Effets des pratiques agroécologiques sur l'efficacité du système productif des producteurs maraîchers au sud du Bénin
}

\author{
Claude Ahouangninou, Docteur \\ Ecole d'Horticulture et d'Aménagement des Espaces Verts (EHAEV) \\ Université Nationale d'Agriculture (UNA), Bénin \\ Centre de Valorisation des Déchets en Energies Renouvelables et en \\ Agriculture (ValDERA), Université d'Abomey-Calavi (UAC), Bénin \\ Institut de Statistiques, Biostatistiques et Sciences Actuarielles \\ Université Catholique de Louvain (UCLouvain), Belgique

\section{Moussa Wari Aboubakar, Docteur}

Centre de Valorisation des Déchets en Energies Renouvelables et en Agriculture (ValDERA), Université d'Abomey-Calavi (UAC), Bénin

\section{Jules Adé, Docteur}

Ecole d'Horticulture et d'Aménagement des Espaces Verts (EHAEV)

Université Nationale d'Agriculture (UNA), Bénin

Keline Meniodem Deliota, Msc

Institut de Statistiques, Biostatistiques et Sciences Actuarielles

Université Catholique de Louvain (UCLouvain), Belgique

\section{Placide Clédjo, Professeur}

Centre de Valorisation des Déchets en Energies Renouvelables et en Agriculture (ValDERA), Université d'Abomey-Calavi (UAC), Bénin

\section{Marie-Paule Kestemont, Professeure}

Institut de Statistiques, Biostatistiques et Sciences Actuarielles, Université Catholique de Louvain (UCLouvain), Belgique

Doi:10.19044/esj.2021.v17n17p267

Submitted: 08 March 2021

Accepted: 06 May 2021

Published: 31 May 2021
Copyright 2021 Author(s)

Under Creative Commons BY-NC-ND

4.0 OPEN ACCESS

Cite As:

Ahouangninou C., Aboubakar M.W., Adé J., Deliota K.M., Clédjo P. \& Kestemont M-P. (2021). Effets des pratiques agroécologiques sur l'efficacité du système productif des producteurs maraîchers au sud du Bénin. European Scientific Journal, ESJ, 17(17), 267. https://doi.org/10.19044/esj.2021.v17n17p267 


\section{Résumé}

La production maraîchère au Bénin est associée à l'usage des intrants chimiques pour améliorer les rendements. Mais face aux risques environnementaux qui pèsent sur le milieu de production, il est nécessaire de produire plus écologique. L'objectif de cette étude est d'évaluer l'effet des pratiques agroécologiques sur l'efficience du système productif des maraîchers. A cet effet, une base de données du Centre VaLDERA renseignant sur les pratiques agricoles, comptes et résultats d'exploitation de 197 producteurs des villes de Cotonou, Sèmè-kpodji et Ouidah en 2014 a été analysée. Une analyse en composante principale (ACP) a permis d'identifier des méta-variables : niveau d'utilisation d'engrais, pratiques agroécologiques et caractéristiques sociodémographiques. Ensuite une regression linéaire multiple log-log a permis d'identifier les variables: quantité de fientes, quantité de NPK utilisées, comme les principaux déterminants de l'efficience des producteurs. Les variables diversité écologique, association culturale, rotation sont faiblement corrélées à l'efficience. Il urge de former les producteurs sur les types de rotation qui puissent améliorer leur niveau de productivité et la qualité de leur production, mais aussi de les sensibiliser à l'usage des engrais organiques pour assurer la durabilité de la production.

Mots clés : Déterminants, efficience, pratiques agroécologiques, maraîchers, Bénin 


\title{
Effects of Agroecological Practices on the Efficiency of the Production System of Vegetable Growers in Southern Benin
}

\author{
Claude Ahouangninou, Docteur \\ Ecole d'Horticulture et d'Aménagement des Espaces Verts (EHAEV) \\ Université Nationale d'Agriculture (UNA), Bénin \\ Centre de Valorisation des Déchets en Energies Renouvelables et en \\ Agriculture (ValDERA), Université d'Abomey-Calavi (UAC), Bénin \\ Institut de Statistiques, Biostatistiques et Sciences Actuarielles \\ Université Catholique de Louvain (UCLouvain), Belgique \\ Moussa Wari Aboubakar, Docteur
}

Centre de Valorisation des Déchets en Energies Renouvelables et en Agriculture (ValDERA), Université d'Abomey-Calavi (UAC), Bénin

\section{Jules Adé, Docteur}

Ecole d'Horticulture et d'Aménagement des Espaces Verts (EHAEV)

Université Nationale d'Agriculture (UNA), Bénin

Keline Meniodem Deliota, Msc

Institut de Statistiques, Biostatistiques et Sciences Actuarielles

Université Catholique de Louvain (UCLouvain), Belgique

Placide Clédjo, Professeur

Centre de Valorisation des Déchets en Energies Renouvelables et en

Agriculture (ValDERA), Université d'Abomey-Calavi (UAC), Bénin

Marie-Paule Kestemont, Professeure

Institut de Statistiques, Biostatistiques et Sciences Actuarielles, Université Catholique de Louvain (UCLouvain), Belgique

\begin{abstract}
Vegetable production in Benin is associated with the use of chemical inputs to improve yields. However, given the environmental risks that weigh on the environment, it is necessary to produce in a more ecological way. The objective of this study is to evaluate the effect of agroecological practices on the efficiency of the market gardeners' production system. To this end, a database from the VaLDERA Centre providing information on the agricultural practices, accounts and operating results of 197 producers in the cities of Cotonou, Sèmè-kpodji and Ouidah in 2014 was analysed. A principal component analysis (PCA) identified meta-variables: level of fertiliser use, agroecological practices and socio-demographic characteristics. Then a multiple log-log linear regression identified variables: amount of manure, amount of NPK used, as the main determinants of producer efficiency. The variables ecological diversity, crop association and rotation are weakly correlated with efficiency. There is an urgent need to train producers on the
\end{abstract}


types of rotation that can improve their level of productivity and the quality of their production, but also to make them aware of the use of organic fertilisers to ensure the sustainability of production.

Keywords: Determinants, efficiency, agro-ecological practices, market gardeners, Benin

\section{Introduction}

La production agricole occupe une place importante dans l'économie du Bénin. Elle a contribué à près de 30,48 \% au Produit Intérieur Brut (PIB) du Bénin sur la période 2005-2008 (PSRSA, 2009; INSAE, 2014). De 2011 à 2014, la contribution moyenne du PIB est de 32,7\% (PSDSA, 2017). La production agricole au Bénin englobe la production végétale, animale et la pêche. La production végétale est la branche la plus importante. Elle a contribué à près de 75\% du PIB agricole sur la période 2011-2014 (PSDSA, 2017). Elle met à disposition des céréales, des tubercules, les légumes et fruits pour la population. La production des légumes et fruits s'est surtout développée dans les villes où la disponibilité en terre se raréfie. Dans ce contexte, les producteurs de fruits et légumes du sud du Bénin intensifient leur système de production en combinant de différentes façons les intrants pour avoir un bon rendement (Ahouangninou et al., 2020. Certains ignorent le volet protection de l'environnement dans la production en utilisant plus d'engrais minéraux (NPK et Urée). L'usage excessive d'engrais minéraux peut entraîner des problèmes de contamination de l'environnement (pollution par les nitrates et nitrites). Dovonou et al. (2017) ont trouvé des teneurs en nitrates, nitrites et phosphates dépassant les normes admises dans les eaux de consommation au Bénin. Certains effets des pratiques agricoles sur la pollution des eaux de surfaces ont été documentés. Il s'agit de la contamination des eaux de surface par les pesticides, les nitrates et les phosphates (Lawani et al., 2017). Des producteurs maraîchers font une association d'engrais minéraux et organiques. Cette dernière pratique s'insère dans les pratiques agroécologiques, levier de la durabilité de la production. La plupart des maraîchers utilise des engrais organiques en association des engrais chimiques (Ahouangninou, 2013). Mais les proportions utilisées peuvent diverger selon les producteurs et les lieux de production. Les producteurs de fruits et légumes font également des associations culturales et de la rotation culturale, mais les associations et rotation effectuées peuvent ne pas avoir une cohérence agronomique dans certains cas.

L'objectif de cette étude est d'évaluer l'effet des pratiques agroécologiques sur l'efficience du système productif des maraîchers au moyen de la modélisation. 


\section{Méthodologie d'étude \\ Collecte des données}

Les données utilisées sont des données transversales collectées par le Centre de Valorisation des déchets en énergies renouvelables et en agriculture (Centre ValDERA) de l'Université d'Abomey-Calavi(UAC) au Sud du Bénin en 2014 sur les sites maraîchers de Cotonou, Sèmè-Kpodji et Ouidah. Les données comportent 197 observations, 11 variables quantitatives et 5 variables qualitatives. La variable efficience est un score sur une échelle de 0 à 25 (Ahouangninou, 2013; Ahouangninou et al., 2016). Ces scores ont été multipliés par 4 pour avoir un score compris entre 0 et 100 . Pour obtenir les scores d'efficience, un ratio du (produits-intrants)/produits (Vilain et al., 2008) a été calculé pour chaque exploitation et les scores ont été attribués suivant une grille d'échelle car le ratio peut être supérieur à 1 (Ahouangninou, 2013; Ahouangninou et al., 2016). Les scores au niveau de assolement, de diversité écologique, de rotation et succession ont été attribués suivant l'échelle de dimension agroécologique de durabilité (Ahouangninou, 2013; Ahouangninou et al., 2016).

\section{Analyses statistiques}

Une Analyse en Composantes Principales (ACP) a été réalisée avec le logiciel R4.1 pour identifier les méta-variables corrélées à des dimensions. Ensuite des régressions linéaires ont été réalisées avec SAS Studio pour identifier les variables influençant l'efficience des producteurs et sélectionner le meilleur modèle permettant de prédire l'efficience dans la production. Le modèle de base utilisé est le suivant :

Efficience $=\beta_{0}+\beta_{1}$ Age $+\beta_{2}$ AnExper $+\beta_{3}$ Assolement $+\beta_{4}$ ConsomNPK_Kg $+\beta_{5}$ Consomuree_Kg $+\beta_{6}$ Consomfiente_Kg $+\beta_{7}$ Divecologique + $\beta_{8}$ Nbre_enfant $+\beta_{9}$ Rotation_succession $+\beta_{10}$ Site $1+\beta_{11}$ Site $2+$ $\beta_{12}$ Niveauinstruction $1+\beta_{13}$ Niveauinstruction $2+\beta_{14}$ Niveauinstruction $3+$ $\beta_{15}$ Sexe $1+\beta_{16}$ Activprin $1+\beta_{17}$ Situationmatrimoniale $1+\varepsilon$

Dix observations ont été choisies de façon aléatoire pour servir d'échantillon test. Les 187 observations restantes ont servi d'échantillon d'apprentissage pour estimer les modèles.

\section{Résultats et Discussion}

\section{Caractéristiques socioéconomiques des enquêtés et exploration des liens entres variables}

Le tableau 1 présente les statistiques descriptives sur les variables quantitatives avec l'échantillon d'apprentissage. L'âge moyen des producteurs est de 36,64 ans. L'âge minimum est 17 ans et le plus âgé a 75 ans. Le nombre 
d'années d'expérience dans la production varie de 1 à 50 ans avec une moyenne de 14,49 ans.

La moyenne de l'efficience des exploitations est de 38,22 unités de point sur cent. La superficie moyenne de terre emblavée est de $1361,27 \mathrm{~m}^{2}$ avec une grande variabilité (écart-type de 2119,50). Pour accroître leur productivité, les producteurs utilisent des engrais chimiques (urée et NPK) et organiques (fientes de volailles). Les quantités moyennes de NPK, urée, fientes utilisées sont respectivement de $228,07 \mathrm{Kg}, 232,36 \mathrm{Kg}$, de $6253,52 \mathrm{Kg}$. Les scores moyens de diversité écologique, assolement, rotation et succession culturale sont respectivement de 11,$45 ; 5,59 ; 4,35$. Ceci dénote d'une

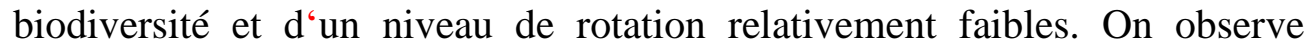
également une faible variabilité de ces scores autour de leurs moyennes (Tableau 1).

Tableau 1. Statistiques descriptives des variables quantitatives

\begin{tabular}{|l|c|c|c|c|}
\hline Variable & Moyenne & Ecart-type & Maximum & Minimum \\
\hline Age & 36.64 & 12.47 & 75.00 & 17.00 \\
An_Exper & 14.49 & 10.26 & 50.00 & 1.00 \\
Div_ecologique & 11.45 & 2.95 & 24.00 & 6.00 \\
Assolement & 5.59 & 1.38 & 10.00 & 2.00 \\
Rotation_succession & 4.35 & 0.93 & 6.00 & 1.00 \\
Efficience & 38.22 & 23.23 & 99.00 & 9.00 \\
Superficie & 1361.27 & 2119.50 & 20000.00 & 64.00 \\
Consom_NPK_Kg & 228.07 & 587.14 & 5350.00 & 1.00 \\
Consom_uree_Kg & 232.36 & 411.10 & 3344.00 & 1.00 \\
Consom_fiente_Kg & 6253.52 & 14769.64 & 133750.00 & 50.00 \\
Nbre_enfant & 2.52 & 2.13 & 11.00 & 0.00 \\
\hline
\end{tabular}

Concernant les variables qualitatives, on observe une grande proportion d'hommes pratiquant la production maraîchère $(87,17 \%)$ contre $12,83 \%$ de femmes (Tableau 2). La grande majorité a la production maraîchère comme activité principale $(86,63 \%)$ et plus de la moitié ont leurs exploitations dans la ville de Cotonou. Environ 1/5 soit 19,25\% d'entre eux sont non instruits. Deux-cinquième soit $43,85 \%$ ont atteint le niveau primaire, $33,33 \%$ ont atteint le niveau d'enseignement secondaire. Seuls 3,74\% ont atteint le niveau supérieur. Pour l'accès à l'eau pour arroser les plants, 35,83\% d'entre eux utilisent les eaux de bas-fonds, 36,9\% utilisent les eaux de forage et le reste des eaux de puits. 
Tableau 2. Statistiques descriptives des variables qualitatives

\begin{tabular}{|l|l|r|r|}
\hline Variables & Modalités & Fréquences & Proportions \\
\hline & Féminin & 24 & 12.83 \\
\hline & Masculin & 163 & 87.17 \\
\hline Situation matrimoniale & Célibataire & 36 & 19.25 \\
\hline & Marie & 151 & 80.75 \\
\hline Activité principale & Non & 25 & 13.37 \\
\hline & Oui & 162 & 86.63 \\
\hline Site & Cotonou & 103 & 55.08 \\
\hline & Ouidah & 38 & 20.32 \\
\hline & Sèmè-Kpodji & 46 & 24.60 \\
\hline Accès à l'eau & Basfonds & 67 & 35.83 \\
\hline & Forage & 69 & 36.90 \\
\hline & Puits & 51 & 27.27 \\
\hline Niveau d'instruction & Non instruit & 36 & 19.25 \\
\hline & Primaire & 82 & 43.85 \\
\hline & Secondaire & 62 & 33.16 \\
\hline & Supérieur & 7 & 3.74 \\
\hline
\end{tabular}

La figure 1 montre que $39,66 \%$ de l'inertie totale est expliquée par la dimension 1 de l'ACP et le premier plan factoriel explique 58,07\% de l'information contenue dans le jeu de données.

Les variables efficience, consommation en urée, consommation en NPK et consommation en fientes sont corrélées au premier plan factoriel. Les variables : âge, année d'expérience quant à elles sont liées à la dimension 2 de l'ACP.

Les liens entre efficience et consommation en urée, NPK et fientes sont évidentes. L'utilisation des engrais dans la production est supposée augmenter la productivité. La productivité est un instrument de l'efficience. Ce sont des concepts voisins, mais la productivité n'est pas l'égal de l'efficience (Sharpe,1995). Les engrais contiennent les éléments nutritifs dont a besoin la plante pour croître. Les engrais minéraux tels que NPK sont essentiels pour la fructification, l'urée pour la croissance de la plante. Quant aux engrais organiques (fientes), leur utilisation permet d'amender le sol et maintenir l'activité biologique dans le sol. Ils permettent de lutter contre la minéralisation excessive. Mais, un bon rendement ne signifie pas qu'une unité de production fait mieux. Pour un même niveau de productivité, si l'unité de production utilise moins de ressources que ses concurrents, elle est plus 
efficiente (Ahouangninou et al., 2020). Ainsi l'efficience mesure le ratio revenus/dépenses.

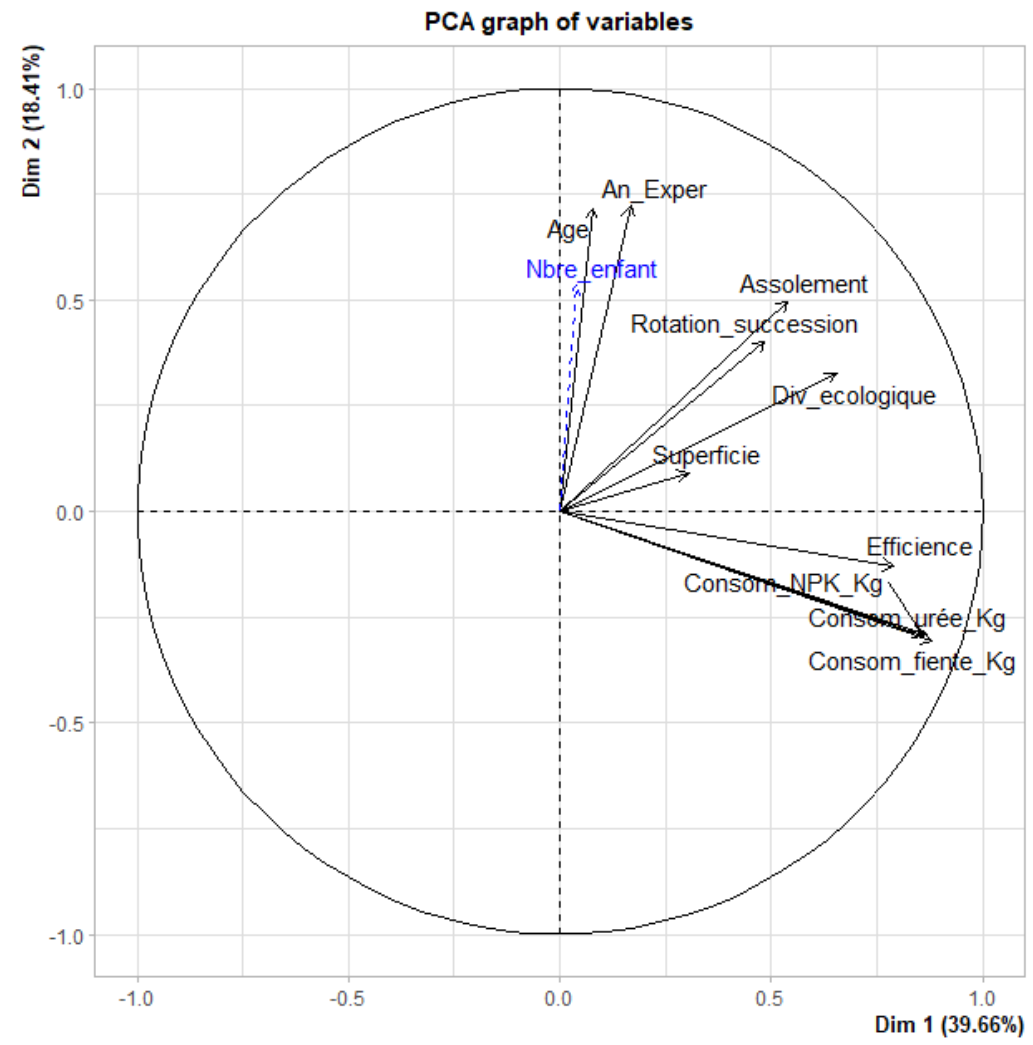

Figure 1 : Analyse en Composantes Principales

\section{Spécification du modèle}

\section{Estimation du Modèle de base déterminant l'efficience}

Le modèle estimé est globalement significatif au seuil de $5 \%(\mathrm{p}<$ 0,001) (Tableau 3). De l'ensemble des coefficients estimés, au moins un est non nul au seuil de 5\%. 59,11\% de la variabilité de l'efficience est expliquée par l'ensemble des variables incluses avec cette spécification linéaire. Les coefficients des variables de consommation en NPK, urée, fiente et Site2 sont significativement différents de zéro. 
Tableau 3. Résultats d'estimation du modèle de base

\begin{tabular}{|c|c|c|c|c|c|c|c|}
\hline \multicolumn{8}{|c|}{ Résultats estimés des paramètres } \\
\hline Variable & DDL & $\begin{array}{l}\text { Valeur estimée } \\
\text { des paramètres }\end{array}$ & $\begin{array}{r}\text { Erreur } \\
\text { type }\end{array}$ & Valeur du test $t$ & $\operatorname{Pr}>|t|$ & Tolérance & $\begin{array}{r}\text { Inflation } \\
\text { de variance }\end{array}$ \\
\hline Intercept & 1 & 5.27631 & 9.28600 & 0.57 & 0.5707 & & 0 \\
\hline Age & 1 & -0.04316 & 0.15936 & -0.27 & 0.7869 & 0.33053 & 3.02546 \\
\hline An_Exper & 1 & 0.25500 & 0.16272 & 1.57 & 0.1189 & 0.46790 & 2.13723 \\
\hline Assolement & 1 & 1.12400 & 1.21399 & 0.93 & 0.3558 & 0.46388 & 2.15575 \\
\hline Consom_NPK_Kg & 1 & -0.01816 & 0.00601 & -3.02 & 0.0029 & 0.10473 & 9.54860 \\
\hline Consom_uree_Kg & 1 & 0.01836 & 0.00631 & 2.91 & 0.0041 & 0.19382 & 5.15934 \\
\hline Consom_fiente_Kg & 1 & 0.00113 & 0.00024308 & 4.63 & $<.0001$ & 0.10126 & 9.87570 \\
\hline Div_ecologique & 1 & -0.12865 & 0.60597 & -0.21 & 0.8321 & 0.40904 & 2.44475 \\
\hline Nbre_enfant & 1 & -0.81599 & 0.82489 & -0.99 & 0.3240 & 0.42442 & 2.35616 \\
\hline Rotation_succession & 1 & 2.71533 & 1.51825 & 1.79 & 0.0755 & 0.64850 & 1.54202 \\
\hline Site1 & 1 & 3.02968 & 3.48373 & 0.87 & 0.3857 & 0.43235 & 2.31293 \\
\hline Site2 & 1 & 11.52504 & 4.50002 & 2.56 & 0.0113 & 0.34565 & 2.89310 \\
\hline Niveau_instruction1 & 1 & 2.90841 & 3.56995 & 0.81 & 0.4164 & 0.41373 & 2.41705 \\
\hline Niveau_instruction2 & 1 & 3.53252 & 3.89576 & 0.91 & 0.3658 & 0.38597 & 2.59086 \\
\hline
\end{tabular}




\begin{tabular}{|l|r|r|r|r|r|r|r|} 
Niveau_instruction3 & 1 & 6.19018 & 7.10541 & 0.87 & 0.3849 & 0.71366 & 1.40122 \\
\hline Sexe1 & 1 & -2.80454 & 3.95585 & -0.71 & 0.4793 & 0.74159 & 1.34846 \\
\hline Activ_prin1 & 1 & 4.81788 & 3.63345 & 1.33 & 0.1866 & 0.84908 & 1.17775 \\
\hline $\begin{array}{l}\text { Situation_matrimoniale } \\
\mathbf{1}\end{array}$ & 1 & 0.23202 & 3.69405 & 0.06 & 0.9500 & 0.61201 & 1.63396 \\
\hline $\mathbf{R}^{2}$ & & 0,5911 & & & & & \\
\hline $\mathbf{R}^{2}$-adj & & 0,550 & & & & & \\
\hline F-value & & 14,37 & & & & & \\
\hline p-value & & $<0.000$ & & & & \\
\hline
\end{tabular}

\section{Test de Ramsey}

Dans le modèle du Reset test de Ramsey, les valeurs prédites du modèle de base ont été récupérées et une regression de la variable réponse Efficience sur l'ensemble des variables explicatives et les valeurs prédites à la puissance $\mathrm{k}$, avec $\mathrm{k}$ allant de 2 à 3 a été réalisée.

L'estimation du modèle montre que le modèle est globalement significatif au seuil de $5 \%$ ( $\mathrm{p}<0,001)$. Ce modèle explique à 85,94\% la variabilité de l'efficience. Dans ce modèle, l'intérêt est porté sur les coefficients de Efficience prédite au carré et au cube. Ces coefficients sont significatifs, ce qui amène à rejeter le modèle de base. Dans la suite un modèle de type log-log sera estimé.

\section{Estimation de modèle fonctionnel}

Le modèle 2 suivant a été estimé:

lEffic $=\beta_{0}+\beta_{1}$ Age $+\beta_{2}$ AnExper $+\beta_{3}$ Assolement $+\beta_{4}$ lNPK $+\beta_{5}$ luree $+\beta_{6}$ lfiente $+\beta_{7}$ Divecologique + $\beta_{8}$ Nbre_enfant $+\beta_{9}$ Rotation_succession $+\beta_{10}$ Site $1+\beta_{11}$ Site $2+\beta_{12}$ Niveauinstruction $1+\beta_{13}$ Niveauinstruction $2+$ $\beta_{14}$ Niveauinstruction $3+\beta_{15}$ Sexe $1+\beta_{16}$ Activprin $1+\beta_{17}$ Situationmatrimoniale $1+\varepsilon$ 
L'estimation du modèle 2 montre qu'il explique 90,58\% de la variabilité du logarithme de l'efficience (Tableau 4). De plus le modèle est globalement significatif ( $p<0,0001)$. Les facteurs d'inflation de la variance (VIF) sont tous en dessous de 10 , ce qui indique une absence de forte colinéarité entre variables explicatives.

Tableau 4. Résultats d'estimation du modèle 2 de type log-log

\begin{tabular}{|c|c|c|c|c|c|c|c|}
\hline \multicolumn{8}{|c|}{ Résultats estimés des paramètres } \\
\hline Variable & DDL & $\begin{array}{r}\text { Valeur estimée } \\
\text { des paramètres }\end{array}$ & $\begin{array}{r}\text { Erreur } \\
\text { type }\end{array}$ & Valeur du test $t$ & $\operatorname{Pr}>|\mathbf{t}|$ & Tolérance & $\begin{array}{l}\text { Inflation } \\
\text { de variance }\end{array}$ \\
\hline Intercept & 1 & 0.39484 & 0.12174 & 3.24 & 0.0014 & $\cdot$ & 0 \\
\hline Age & 1 & -0.00089236 & 0.00175 & -0.51 & 0.6109 & 0.32749 & 3.05354 \\
\hline An_Exper & 1 & 0.00099617 & 0.00179 & 0.56 & 0.5790 & 0.46135 & 2.16755 \\
\hline Assolement & 1 & 0.00417 & 0.01330 & 0.31 & 0.7540 & 0.46217 & 2.16370 \\
\hline Lnpk & 1 & 0.02454 & 0.01340 & 1.83 & 0.0688 & 0.28757 & 3.47743 \\
\hline Lfiente & 1 & 0.38759 & 0.01624 & 23.86 & $<.0001$ & 0.39388 & 2.53882 \\
\hline Luree & 1 & 0.00238 & 0.01879 & 0.13 & 0.8992 & 0.29678 & 3.36944 \\
\hline Div_ecologique & 1 & 0.00178 & 0.00659 & 0.27 & 0.7876 & 0.41425 & 2.41400 \\
\hline Nbre_enfant & 1 & -0.00783 & 0.00902 & -0.87 & 0.3866 & 0.42481 & 2.35398 \\
\hline Rotation_succession & 1 & -0.00238 & 0.01683 & -0.14 & 0.8878 & 0.63083 & 1.58522 \\
\hline Site1 & 1 & 0.05478 & 0.03821 & 1.43 & 0.1536 & 0.42978 & 2.32678 \\
\hline Site2 & 1 & -0.01046 & 0.05247 & -0.20 & 0.8422 & 0.30408 & 3.28862 \\
\hline Niveau_instruction1 & 1 & -0.01357 & 0.03862 & -0.35 & 0.7257 & 0.42277 & 2.36536 \\
\hline Niveau_instruction2 & 1 & -0.05609 & 0.04307 & -1.30 & 0.1946 & 0.37766 & 2.64786 \\
\hline Niveau_instruction3 & 1 & 0.00241 & 0.07676 & 0.03 & 0.9749 & 0.73126 & 1.36750 \\
\hline Sexe1 & 1 & 0.02946 & 0.04357 & 0.68 & 0.5000 & 0.73099 & 1.36801 \\
\hline Activ_prin1 & 1 & -0.03239 & 0.04070 & -0.80 & 0.4273 & 0.80920 & 1.23579 \\
\hline $\begin{array}{l}\text { Situation_matrimoniale } \\
1\end{array}$ & 1 & -0.00650 & 0.04025 & -0.16 & 0.8718 & 0.61640 & 1.62232 \\
\hline $\mathbf{R}^{2}$ & & 0,9058 & & & & & \\
\hline $\mathbf{R}^{2}$-adj & & 0,8963 & & & & & \\
\hline
\end{tabular}




\begin{tabular}{|l|r|r|r|r|l|l|}
\hline F-value & & 95,61 & & & & \\
\hline p-value & & $<0.000$ & & & & \\
\hline
\end{tabular}

Plusieurs coefficients ne sont pas significatifs. En éliminant les coefficients dont la probabilité du test de student est supérieur à $\mathrm{p}=0,3$ et après vérification des critères da Mallows, on obtient le modèle 3 suivant: $\mathbf{I E f f i c}=\boldsymbol{\beta}_{\mathbf{0}}+\boldsymbol{\beta}_{\mathbf{1}} \mathbf{I N P K}+$ $\beta_{2}$ Ifiente $+\beta_{3}$ Site1 $+\beta_{4}$ Niveauinstruction $2+\varepsilon$

De l'ensemble des méthodes de sélection ascendante, descendante et pas-à-pas, les variables communes retenues sont celles de la méthode descendante avec les variables INPK, lfiente et Site1. Ce sont celles qui sont retenues dans le modèle final. Ces variables sont incluses dans les sous-ensembles de variables retenus par les critères de Mallows (Tableau 5).

Modèle final: IEffic $=\beta_{0}+\beta_{1}$ INPK $+\beta_{2}$ Ifiente $+\beta_{3}$ Site1 $+\varepsilon$ 
Tableau 5. Sélection avec Critères de Mallows, $\mathrm{R}^{2}$ et $\mathrm{R}^{2}$-adj du modèle 3

\begin{tabular}{|r|r|r|r|l|}
\hline $\begin{array}{r}\text { Nombre dans } \\
\text { le modèle }\end{array}$ & $\mathbf{C}(\mathbf{p})$ & $\mathbf{R}$ carré & $\begin{array}{r}\text { R carré } \\
\text { ajusté }\end{array}$ & \\
\hline $\mathbf{3}$ & 4.4928 & 0.9021 & 0.9005 & INPK lfiente Site1 \\
\hline $\mathbf{4}$ & 5.0000 & 0.9029 & 0.9007 & lNPK lfiente Site1 Niveau_instruction2 \\
\hline $\mathbf{2}$ & 7.6259 & 0.8993 & 0.8982 & INPK lfiente \\
\hline $\mathbf{3}$ & 8.8786 & 0.8997 & 0.8981 & INPK lfiente Niveau_instruction2 \\
\hline $\mathbf{2}$ & 9.3799 & 0.8984 & 0.8973 & lfiente Site1 \\
\hline $\mathbf{3}$ & 10.2193 & 0.8990 & 0.8974 & lfiente Site1 Niveau_instruction2 \\
\hline $\mathbf{1}$ & 12.0828 & 0.8959 & 0.8953 & Lfiente \\
\hline $\mathbf{2}$ & 13.5333 & 0.8962 & 0.8950 & lfiente Niveau_instruction2 \\
\hline $\mathbf{2}$ & 867.0435 & 0.4406 & 0.4345 & lNPK Niveau_instruction2 \\
\hline $\mathbf{3}$ & 869.0261 & 0.4406 & 0.4315 & INPK Site1 Niveau_instruction2 \\
\hline $\mathbf{1}$ & 874.8964 & 0.4354 & 0.4323 & Lnpk \\
\hline $\mathbf{2}$ & 876.7643 & 0.4354 & 0.4293 & lNPK Site1 \\
\hline $\mathbf{2}$ & 1670.326 & 0.0119 & 0.0012 & Site1 Niveau_instruction2 \\
\hline $\mathbf{1}$ & 1675.308 & 0.0082 & 0.0028 & Site1 \\
\hline $\mathbf{1}$ & 1680.226 & 0.0055 & 0.0002 & Niveau_instruction2 \\
\hline & & & & \\
\hline
\end{tabular}

Le modèle final estimé avec la méthode MCO est globalement significatif et explique à 90,21\% la variabilité de l'efficience. Les indices de conditionnement et les Facteurs d'inflation de variance sont inférieures à 10, ce qui traduit une absence de multicolinéarité.

Les tests de Kolmogorov-Smirnov et test de Shapiro-Wilk confirment que les résidus suivent une loi normale (Tableau 6). Le QQ-plot et l'allure de l'histogramme des résidus confirment bien la normalité des résidus du modèle (Figures 2). Mais les tests de White (Tableau 7) et de Breusch-Pagan (Tableau 8) concluent à un hétéroscédasticité des erreurs avec le modèle final estimé par Moindre Carrés Ordinaires (MCO). 
Tableau 6. Test de Normalité des résidus

\begin{tabular}{|l|l|r|l|r|}
\hline \multicolumn{5}{|c|}{ Tests de normalité } \\
\hline Test & \multicolumn{2}{|c|}{ Statistique } & \multicolumn{2}{c|}{ p-value } \\
\hline Shapiro-Wilk & W & 0.994044 & Pr $<$ W & 0.6572 \\
\hline Kolmogorov-Smirnov & D & 0.0516 & Pr $>$ D & $>0.1500$ \\
\hline Cramer-von Mises & W-Sq & 0.047907 & Pr $>$ W-Sq & $>0.2500$ \\
\hline Anderson-Darling & A-Sq & 0.295301 & Pr $>$ A-Sq & $>0.2500$ \\
\hline
\end{tabular}
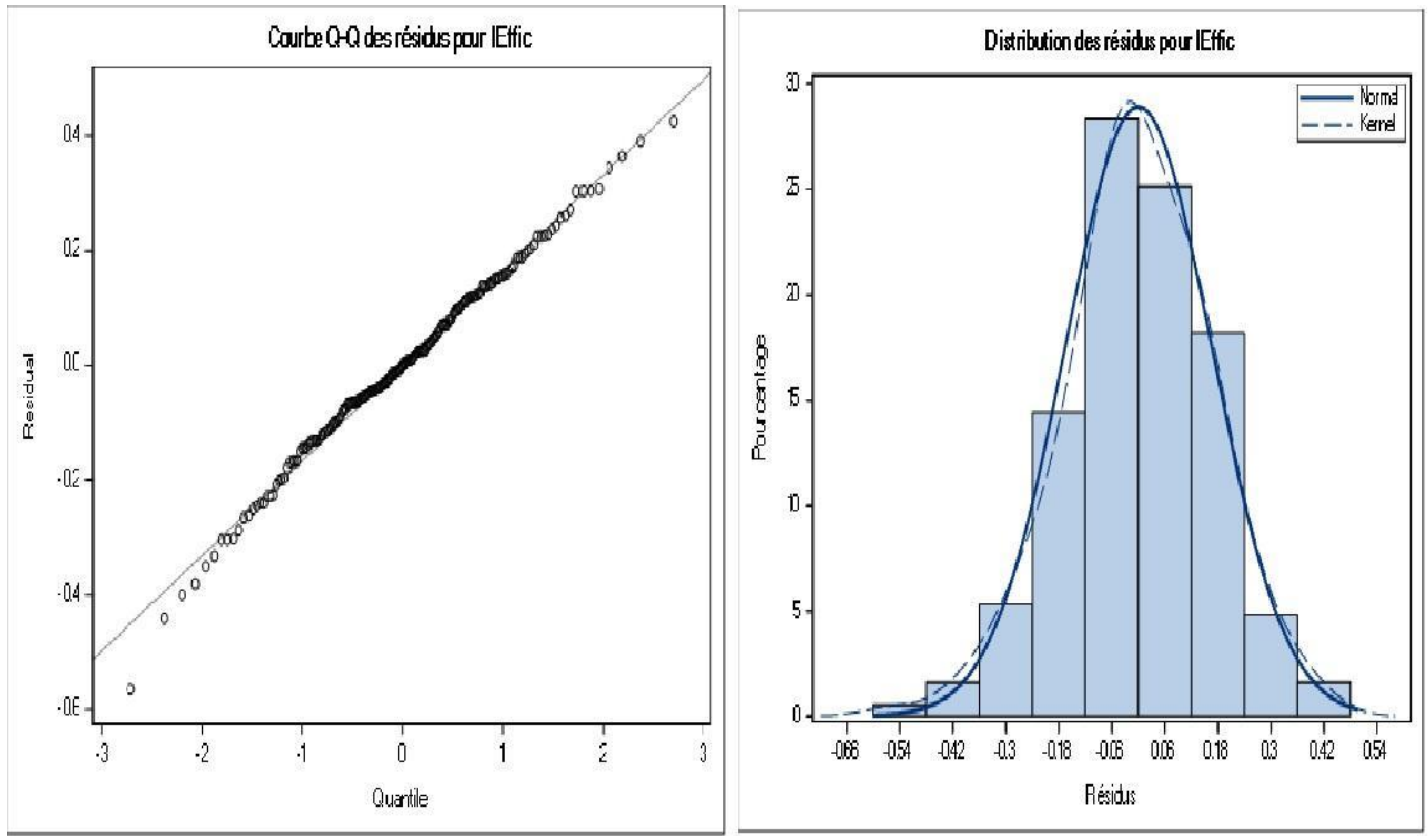

Figure 2. QQ-plot et Histogramme des résidus 
Tableau 7. Résultats de l'estimation du Test de White

\begin{tabular}{|l|r|r|r|r|r|}
\hline \multicolumn{7}{|c|}{ Résultats estimés des paramètres } \\
\hline Variable & DDL & $\begin{array}{r}\text { Valeur estimée } \\
\text { des paramètres }\end{array}$ & $\begin{array}{r}\text { Erreur } \\
\text { type }\end{array}$ & Valeur du test t & Pr > |t $\mid$ \\
\hline Intercept & 1 & 0.19641 & 0.09468 & 2.07 & 0.0395 \\
\hline Lnpk & 1 & -0.02201 & 0.01165 & -1.89 & 0.0605 \\
\hline Lfiente & 1 & -0.04320 & 0.02440 & -1.77 & 0.0784 \\
\hline Site1 & 1 & 0.12000 & 0.03954 & 3.03 & 0.0028 \\
\hline Ifiente2 & 1 & 0.00262 & 0.00163 & 1.61 & 0.1103 \\
\hline INPK2 & 1 & 0.00008819 & 0.00092651 & 0.10 & 0.9243 \\
\hline LNPKIfiente & 1 & 0.00282 & 0.00150 & 1.88 & 0.0612 \\
\hline INPKSITE1 & 1 & 0.00553 & 0.00414 & 1.34 & 0.1834 \\
\hline IfienteSITE1 & 1 & -0.01868 & 0.00611 & -3.06 & 0.0026 \\
\hline $\mathbf{R}^{\mathbf{2}}$ & & 0.2748 & & & \\
\hline $\mathbf{R}^{2}$-adj & & 0.2422 & & & \\
\hline F-value & & 8.43 & & & \\
\hline p-value & & $<.0001$ & & & \\
\hline
\end{tabular}

Tableau 8. Résultats de l'estimation de Test de Breusch-Pagan

\begin{tabular}{|l|r|r|r|r|r|}
\hline \multicolumn{7}{|c|}{ Résultats estimés des paramètres } \\
\hline Variable & DDL & $\begin{array}{r}\text { Valeur estimée } \\
\text { des paramètres }\end{array}$ & $\begin{array}{r}\text { Erreur } \\
\text { type }\end{array}$ & Valeur du test t & Pr > $|\mathbf{t}|$ \\
\hline Intercept & 1 & -0.03639 & 0.02063 & -1.76 & 0.0795 \\
\hline Lnpk & 1 & 0.00503 & 0.00219 & 2.29 & 0.0230 \\
\hline Lfiente & 1 & 0.00611 & 0.00312 & 1.96 & 0.0520 \\
\hline Site1 & 1 & -0.00598 & 0.00588 & -1.02 & 0.3103 \\
\hline $\mathbf{R}^{\mathbf{2}}$ & & 0.1349 & & & \\
\hline $\mathbf{R}^{2}$-adj & & 0.1208 & & & \\
\hline F-value & & 9.52 & & & \\
\hline p-value & & $<.0001$ & & & \\
\hline
\end{tabular}

Le modèle estimé par la méthode Robuste fournit les meilleures estimations car prend en compte l'hétéroscédasticité des erreurs dans l'estimation des paramètres (Figure 3). 


\begin{tabular}{|l|r|r|lr|r|}
\hline \multicolumn{7}{|c|}{ Résultats estimés des paramètres } \\
\hline Variable & DDL & $\begin{array}{r}\text { Valeur estimée } \\
\text { des paramètres }\end{array}$ & $\begin{array}{r}\text { Erreur } \\
\text { type Valeur du test t }\end{array}$ & Pr > t t $\mid$ \\
Intercept & 1 & 0.35025 & 0.08718 & 4.02 & $<.0001$ \\
INPK & 1 & 0.02430 & 0.00927 & 2.62 & 0.0095 \\
Ifiente & 1 & 0.38971 & 0.01320 & 29.53 & $<.0001$ \\
Site1 & 1 & 0.05620 & 0.02484 & 2.26 & 0.0248 \\
\hline
\end{tabular}

\begin{tabular}{|l|r|rrr|rr|r|}
\hline \multicolumn{7}{|c|}{ Résultats estimés des paramètres } \\
\hline Paramètre & DDL & Estimation & $\begin{array}{c}\text { Erreur } \\
\text { type }\end{array}$ & $\begin{array}{c}\text { Intervalle de } \\
\text { confiance } \\
\text { à95\% }\end{array}$ & Khi-2 & Pr > khi-2 \\
Intercept & 1 & 0.2258 & 0.0857 & 0.0578 & 0.3939 & 6.94 & 0.0085 \\
INPK & 1 & 0.0272 & 0.0091 & 0.0094 & 0.0451 & 8.93 & 0.0028 \\
Ifiente & 1 & 0.4059 & 0.0130 & 0.3804 & 0.4313 & 977.64 & $<.0001$ \\
Site1 & 1 & 0.0478 & 0.0244 & -0.0001 & 0.0957 & 3.83 & 0.0503 \\
Scale & 1 & 0.1540 & & & & & \\
\hline
\end{tabular}

Figure 3. Résultats d'estimation du modèle final par MCO ( Tableau de haut) et Robuste (Tableau du bas)

\section{Significativité et Interprétation}

Le modèle final estimé par MCO est globalement significatif au seuil de $1 \%$ (Figure 3). L'ensemble des regresseurs ont leurs coefficients qui sont significativement différents de zéro au seuil de 5\%. Un pourcent (1\%) d'augmentation de quantité consommée d'engrais NPK augmente l'efficience dans la production de $0,024 \%$ alors que l'augmentation de $1 \%$ d'utilisation d'engrais organique fiente augmente de $0,39 \%$ l'efficience du système productif, ceteris paribus. Les producteurs de Cotonou ont une efficience de $5,62 \%$ supérieure à celles des deux autres sites, toutes les autres variables étant maintenues fixes. Mais du fait de l'hétéroscédasticité des erreurs avec cette méthode, la méthode robuste est préférée.

Avec la méthode robuste, une augmentation de $1 \%$ de quantité d'engrais NPK entraîne un accroissement de 0,027\% de l'efficience dans la production toutes les autres variables étant maintenues fixes, alors que $1 \%$ d'augmentation de fiente entraîne une augmentation de $0,406 \%$ de l'efficience (Figure 3). Les producteurs de Cotonou ont une efficience de 4,78\% supérieure à celles des autres producteurs toute chose étant par ailleurs égale. L'utilisation d'engrais chimique NPK influence aussi l'efficience des producteurs. Une augmentation de 1\% d'utilisation de NPK entraîne 0,027\% d'augmentation de l'efficience du système tout étant par ailleurs égal. Mais, un usage excessif de NPK peut avoir un effet négatif sur la productivité, car il 
peut devenir toxique pour la plante. D'autre part, cet excès peut entraîner des pollutions de l'environnement (Dovonou et al., 2017 ; Lawani et al., 2017 ; Mondé et al., 2020). Pour une durabilité des systèmes de production maraîchers, le recours aux intrants chimiques doit être limité en mettant plus d'accent sur l'usage des engrais organiques (Ahouangninou et al., 2016 ; Ouédraogo et al., 2020). L'usage d'engrais organiques dans le système de production donne des rendements meilleurs à l'agriculture conventionnelle (Sardana et al., 2013 ; Bruelle et al., 2014). Gosh et Devi (2018) trouvent que l'incorporation du compost dans la fertilisation accroît les rendements du riz de $13,61 \%$. Les engrais organiques sont riches en nutriments pour la plante (Singh et al., 2019). Les fientes de volaille (poulet) contiennent d'importantes quantités d'azote totale, de carbone total, de potassium, de calcium. Singh et al. (2018) ont conclu que le compostage améliore les qualités nutritives des fientes de volailles en augmentant les teneurs en carbone totale, potassium, sodium, calcium, magnésium ainsi qu'en énergie totale contenue. De plus l'amendement organique a d'effets positifs sur les propriétés du sol (Mieldazys et al., 2019). Cogger (2005) rapporte que l'usage du compost permet la restauration des sols dégradés. La présence de matières organiques va favoriser le développement de vers de terre et d'autres organismes bénéfiques pour la croissance de la plante. Al-Bataina et al. (2016) trouvent un effet significatif de l'âge du compost sur la libération des nutriments. Utiliser du compost non mûr peut être préjudiciable pour la plante car le compost non mûr va plus libérer d'azote qui peut être toxique pour les jeunes plants et compromettre les objectifs de performance du producteur.

\section{Prévisions du modèle}

A travers les diagnostics du modèle, le modèle final est adéquat et peut servir à faire des prévisions. Des intervalles de confiance $\left(\mathrm{IC}_{95}\right)$ de valeurs prédites pour de nouvelles observations ont été faites pour la méthode $\mathrm{MCO}$ et la méthode Robuste. Les estimations des deux méthodes sont proches, mais l'erreur standard et l'intervalle de confiance pour la méthode robuste sont un peu plus larges. Le tableau 9 montre les prévisions du logarithme de $1^{\prime}$ efficience (lEffic) pour les 10 observations éliminées au départ (échantillon test) respectivement par MCO et la méthode Robuste. Une observation du tableau révèle que les deux méthodes donnent des prévisions proches.

L'intervalle de confiance moyen de prédiction par la méthode Robuste est $[3,1944 ; 3,8055]$. La méthode Robuste peut faire des estimations de lEffic entre $[1,616 ; 5,564]$ (Tableau 10). Cet intervalle de confiance (IC95) couvre bien les valeurs réelles de lEffic pour les 10 observations exclues au départ. 
Tableau 9. Prévisions par MCO et Robuste pour les 10 obs exclues au départ

\begin{tabular}{|r|c|c|c|}
\hline Obs. & IEffic & Ypred (Robuste) & Ypred (MCO) \\
\hline $\mathbf{1}$ & 2.70805 & 2.89645 & 2.91798 \\
\hline $\mathbf{2}$ & 2.48491 & 2.77919 & 2.80719 \\
\hline $\mathbf{3}$ & 3.80666 & 3.82704 & 3.79823 \\
\hline $\mathbf{4}$ & 3.95124 & 3.97277 & 3.93940 \\
\hline $\mathbf{5}$ & 3.33220 & 3.40352 & 3.39505 \\
\hline $\mathbf{6}$ & 3.21888 & 3.09229 & 3.09822 \\
\hline $\mathbf{7}$ & 3.33220 & 3.19122 & 3.19154 \\
$\mathbf{8}$ & 3.33220 & 3.22370 & 3.22274 \\
\hline $\mathbf{9}$ & 4.00733 & 3.77048 & 3.74770 \\
\hline $\mathbf{1 0}$ & 3.46574 & 3.41741 & 3.40746 \\
\hline
\end{tabular}

Tableau 10. Intervalles de Confiance de Prévisions par méthode Robuste

\begin{tabular}{|c|c|c|c|}
\hline Variable & Moyenne & Minimum & Maximum \\
\hline IEffic (logarithme efficience) & 3.4923814 & 2.1972246 & 4.5951199 \\
Ypred (prediction) & 3.4999339 & 1.9390594 & 5.2505192 \\
ICLow95 & 3.1943993 & 1.6165577 & 4.9370018 \\
ICUpp95 & 3.8054685 & 2.2615610 & 5.5640366 \\
\hline
\end{tabular}

\section{Conclusion}

Afin d'identifier les variables déterminant l'efficience dans la production des maraîchers et de fournir un modèle permettant de le prédire, une analyse en composantes principales (ACP) et une regression de type log$\log$ ont été utilisées. Les résultats montrent que le niveau d'utilisation d'engrais et l'efficience sont corrélées à la première dimension de l'ACP. Le modèle de regression linéaire multiple log-log retenu a permis d'identifier les variables: quantité de fientes, quantité de NPK utilisées et le site de production, comme les principaux déterminants de l'efficience des producteurs. Le modèle final estimé avec le modèle robuste explique à 90,21\% de la variabilité de l'efficience et respecte les hypothèses de normalité des résidus, hypothèse de résidus de moyenne nulle, et absence de multicolinéarité. La variable rotation et succession culturale est également corrélée à l'efficience. Il urge de sensibiliser les producteurs sur l'usage des engrais organiques dans l'amendement des sols, mais aussi à l'utilisation de bonnes pratiques de rotation culturale pour garantir l'efficience et assurer la durabilité de la production.

\section{References:}

1. Ahouangninou C. (2013). Durabilité de la production maraîchère au sud-Bénin : un essai de l'approche écosystémique. Thèse de Doctorat 
Unique en Gestion de l'Environnement, EDP, FLASH, Université d'Abomey-Calavi, Bénin, 2013, 358 p.

2. Ahouangninou C., Cledjo P., Martin T., Assogba-Komlan F., Kpenavoun S., Nouatin G., Boko W., Soumanou M., Houssou C., Biaou G., Ahanchede A., Boko M., \& Fayomi B (2016). Evaluation de la durabilité de la production maraîchère au sud du Bénin. Afrique Science, 12 (1) : 119-134.

3. Ahouangninou C., Boko S.Y.W., Arouna A, Logbo J, Fayomi B, \& Martin T. (2020). Performance environnementale et économique dans la production de la grande morelle (Solanum macrocarpon) au sud du Bénin: une évaluation des efficacités technique, allocative et économique. Agronomie Africaine, 32(2) : 135-149

4. Al-Bataina B.B., Young T.M., \& Ranieri E. (2016). Effects of compost age on the release of nutrients. Int. Soil Water Conserv Res 4(3):230 236

5. Bruelle G., Naudin K., Scopel E., Domas R., Rabeharisoa L., \& Tittonel P.A. (2014). Short- to mid- term impact of conservation agriculture on yield variability of upland rice: Evidence from farmer's fields in Madagascar. Experimental Agriculture, 51(1) : 66-84

6. Cogger C. (2005). Bénéfices potentiels du compost pour la restauration des sols perturbés par le développement urbain. Science et utilisation du compost 13 (4) 243-251

7. Dovonou F.E., Alassane A., Adjahossou V.N., Agbodo B., Djibril R., \& Mama D. (2017). Impacts de l'assainissement autonome sur la qualité des eaux de puits dans la commune de Sèmè-kpodji (SudBénin). Int. J. Biol. Chem. Sci, 11(6): 3086-3099

8. Ghosh M., \& Devi A. (2019). Assessment of crop growth, soil properties and crop yield in an upland acidic soil with inorganic fertilizer blended with organic amendments in summer rice cropping seasons. International Journal of Recycling of Organic Waste in Agriculture 8 (Suppl 1):S1-S9

9. INSAE (2014). Statistiques économiques : Production agricole, République du Bénin, (2014)

10. Lawani RAN., Kelomè N.C., Agassounon Djikpo Tchibozo M., Hounkpè J.B., Adjagodo A. (2017). Effets des pratiques agricoles sur la pollution des eaux de surface en République du Bénin, Larhyss Journal, $30: 173-190$

11. Mieldazys R., Jotautienè E., Jasinskas A., Pekarskas J., Zinkevicienė R. (2019). Investigation of physical-mechanical properties and impact on soil of granulated manure compost fertilizers. Journal of Environmental Engineering and Landscape Management, 27 (3): 153162 
12. Mondé Fifatin M-A., Ahouangninou C., Ayena A.A, Massede S., Tente B. (2020). Analyse de la gestion des intrants chimiques dans la commune de Tori-Bossito au Bénin. International Journal of Progressive Sciences and Technologies, 22(2) : 60-71

13. Ouédraogo F., Ahouangninou C., Kestemont M-P., \& Kaboré Konkobo M. (2020). Evaluation de la durabilité des exploitations maraîchères du Burkina Faso suivant une approche écosociosystémique (cas de la province du Houet). Tropicultura, 38(2, 1520) : 2295-8010

14. PSDSA (2017). Plan Stratégique de Développement du Secteur Agricole au Bénin, République du Bénin

15. PSRSA (2009). Plan Stratégique de Relance du Secteur Agricole au Bénin, 173 p.

16. Sardana H., Bhat M.N, \& Sehgal M. (2013). Evaluation and validation of non-chemical IPM and inm technology for bell pepper (Capsicum annum var. Frutescens L.) through farmers' participatory approach. International Journal of Entomology, 75(2) : 127-131

17. Sharpe A. (1995). International perspectives on productivity and efficiency, Review of income and Wealth, 1995, 41(2): 221-237.

18. Singh G., Shamsuddin M.R, Aqsha A., \& Lim S.W. (2018). Characterization of Chicken Manure from Manjung Region. IOP Conf. Series: Materials Science and Engineering 458 (2018) 012084 doi:10.1088/1757-899X/458/1/012084

19. Vilain L., Boisset K., Girardin P., Guillaumin A., Mouchet C, Viaux P., \& Zahm P. (2008). La méthode IDEA, Guide d'utilisation, Troisième édition Educagri, Dijon, France, 159 p. 